\title{
Development of a compact neutron source based on field ionization processes
}

\author{
Arun Persaud,* Ian Allen, Michael R. Dickinson, and Thomas Schenkel \\ E.O. Lawrence Berkeley National Laboratory \\ Berkeley, CA 94720, USA \\ Rehan Kapadia, Kuniharu Takei, and Ali Javey \\ Department of Electrical Engineering and Computer Sciences \\ University of California at Berkeley \\ Berkeley, CA 94720, USA
}

\begin{abstract}
The authors report on the use of carbon nanofiber nanoemitters to ionize deuterium atoms for the generation of neutrons in a deuterium-deuterium reaction in a preloaded target. Acceleration voltages in the range of $50-80 \mathrm{kV}$ are used. Field emission of electrons is investigated to characterize the emitters. The experimental setup and sample preparation are described and first data of neutron production are presented. Ongoing experiments to increase neutron production yields by optimizing the field emitter geometry and surface conditions are discussed.
\end{abstract}

PACS numbers: 29.25.Dz; 81.07.De; 79.70.+q

\section{INTRODUCTION}

In recent years, the use of nanoemitters for neutron production has been investigated and progress on several approaches such as field desorption ${ }^{1}$ and field evaporation sources $^{2}$ has been reported. In addition, there have been investigations into the use of field ionization for neutron production $^{3,4}$.

For field ionization, a strong electric field generated by, e.g., a sharp tip in combination with the Coulomb potential of an atom (or molecule), provides a tunnel barrier that is small enough to allow an electron from the atom to tunnel into the tip and thereby ionize the atom. Field strengths of the order of $20 \mathrm{~V} / \mathrm{nm}$ are needed. Field desorption and field evaporation rely on a layer of adsorbed material or the tip material itself, and require higher fields than those required for field ionization $(>$ $40 \mathrm{~V} / \mathrm{nm}$ ). For comparison, electron field emission requires fields of the order of $2 \mathrm{~V} / \mathrm{nm}^{5}$.

A device using nanoemitters can achieve a compact and inexpensive source with a low energy budget compared to rf-plasma or Penning sources and is therefore a candidate for a portable sealed source that can be used in oil-well logging or more generally as a replacement for radioactive sources. Our approach relies on the use of nanoemitters that are relatively easy to fabricate.

To generate neutrons, we utilize the $\mathrm{D}(\mathrm{d}, \mathrm{n})^{3} \mathrm{He}$ reaction by accelerating deuterium ions onto a stationary, deuterated titanium target. Acceleration voltages of 50$80 \mathrm{kV}$ are needed to achieve neutron yields in the $10^{8} \mathrm{n} / \mathrm{s}$ range ${ }^{6}$. We also use this voltage to directly apply the necessary fields to the emitters by placing the target electrode at an appropriate distance.

\section{SAMPLES}

To generate the high fields needed for field ionization, we make use of the fact that a sharp tip, e.g., a single carbon nanofiber $(\mathrm{CNF})$, in an electric field compresses the field lines generating fields that are several thousand times stronger than the field gradient in a parallel capacitor geometry. For an illustration of the enhancement effect, see Fig. 1, which shows the simulation results of two sharp tips disturbing the field between two plates. The simulation was done in Warp3D ${ }^{7}$.



FIG. 1. Simulations of field enhancement by an array of high aspect ratio tips (arbitrary size).

Field enhancement allows us to generate the desired field of several Volts per Angstrom at the tip by applying our acceleration voltage over a distance of a few centimeters.

For the results reported in this article, we use a sample of aligned multiwall CNFs grown on a silicon wafer in 
a plasma-enhanced chemical vapor deposition (PEVCD) process $^{8}$. A copper layer of $200 \mathrm{~nm}$ is deposited on a $50 \mathrm{~nm}$ oxide layer of a silicon wafer, followed by a $30 \mathrm{~nm}$ titanium layer. Then, a final $30 \mathrm{~nm}$ layer of nickel is deposited. The nickel layer is used as a catalyst for the CNF growth and the titanium layer serves as a diffusion barrier for the nickel during the PEVCD process. The growth process uses $\mathrm{NH}_{3}$ and $\mathrm{C}_{2} \mathrm{H}_{2}$ gases in a dc plasma at $900^{\circ} \mathrm{C}$. A scanning electron microscope (SEM) image of the CNFs is shown in Fig. 2. The diameter of the CNFs is about $70 \mathrm{~nm}$. As can be seen in the figure, the growth results in a relatively uniform height, but single nanofibers also extend above the forest. These will show the highest enhancement factors.

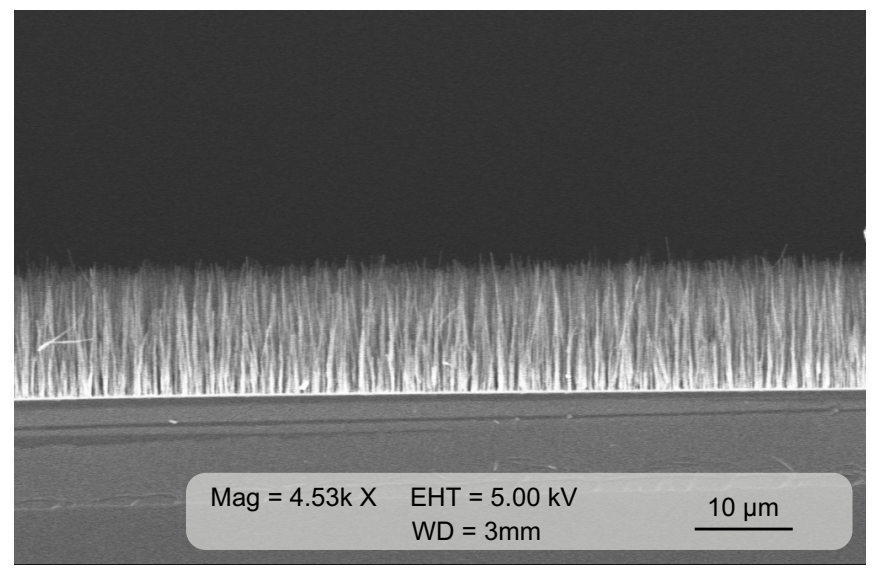

FIG. 2. SEM image showing cross section of aligned carbon nanofibers grown on a silicon substrate.

\section{SETUP}

The experimental setup consists of a vacuum system (base pressure $10^{-5} \mathrm{~Pa}$ ) featuring two electrodes in a parallel plate arrangement with the possibility to vary the distance between the plates. One of the electrodes, the target, can be biased to a high negative voltage (up to $80 \mathrm{kV}$ ) and features a several millimeters thick titanium surface that is used to preload the target with deuterium in a separate process. The other electrode is normally grounded through a current meter and is used to mount the nanoemitters. The emitters are exposed to deuterium molecules or deuterium atoms from a Mantis MGC75 gas cracker source that can produce a beam of neutral deuterium with a high atomic fraction $(\sim 80 \%)$. This is advantageous since accelerated atomic ions will have a higher kinetic energy per nucleus compared to molecular ions which translates to a higher D-D reaction cross section yielding a factor of ten higher neutron flux. From Csikai's work ${ }^{6}$, we can expect $10^{5} \mathrm{n} / \mathrm{s} / \mu \mathrm{A}$ for $80 \mathrm{keV}$ deuterium ions and $10^{4} \mathrm{n} / \mathrm{s} / \mu \mathrm{A}$ for $40 \mathrm{keV}$ ions using a fully loaded target (i.e., $\mathrm{Ti}_{1} \mathrm{D}_{2}$ ).

The neutrons are detected using a Health Physics In- struments Model 6060 neutron detector. We calibrated the detector using AmBe and PuBe sources of known activity in order to be able to convert from $\mathrm{mRem} / \mathrm{h}$ to absolute values of $\mathrm{n} / \mathrm{s}$. The background level was measured over a time frame of several days to 0.5 counts $/ \mathrm{min}$.

The setup also allows us to run the emitters in electron emission mode by positively biasing the target. We make use of this to characterize the emitters and to evaluate the field enhancement factor, which can be obtained by standard Fowler-Nordheim analysis.

\section{RESULTS}

Field emission studies at a emitter-target distance of $25.4 \mathrm{~mm}$ and a pressure of $10^{-5} \mathrm{~Pa}$ show onset fields for electron emission in the range of $10^{6} \mathrm{~V} / \mathrm{m}$, see Fig. 3 . The insert in Fig. 3 shows a Fowler-Nordheim plot that is a plot of the inverse electric field versus $\log \left(I / E^{2}\right)$. For field emission, a linear relation between these two quantities as in Eq. (1) is predicted ${ }^{9}$ and fits our data well. The Fowler-Nordheim equation used to fit the data is

$$
\log \left(\frac{I}{E^{2}}\right)=\log \left(\frac{c_{a} A \gamma^{2}}{\Phi}\right)-\frac{c_{b} \Phi^{3 / 2}}{\gamma} \frac{1}{E},
$$

where $I(\mathrm{~A})$ the emitted current, $E(\mathrm{~V} / \mathrm{m})$ the applied field, $\gamma$ the field enhancement factor (so that $\gamma E$ is the local field at the tip), $A\left(\mathrm{~m}^{2}\right)$ the area of the emitter, $\Phi(\mathrm{eV})$ the work function of the material $(4.8 \mathrm{eV}$ was assumed for $\mathrm{CNF}), c_{a}=1.5414 \times 10^{-6} \mathrm{~A} \cdot \mathrm{eV} \cdot \mathrm{V}^{-2}$ and $c_{b}=6.8309 \times 10^{9} \mathrm{~V} \cdot \mathrm{m} \cdot \mathrm{eV}^{3 / 2}$. From the slope of the linear fit, the field enhancement factor can be calculated. Our measurements give an enhancement factor of around 5000 .

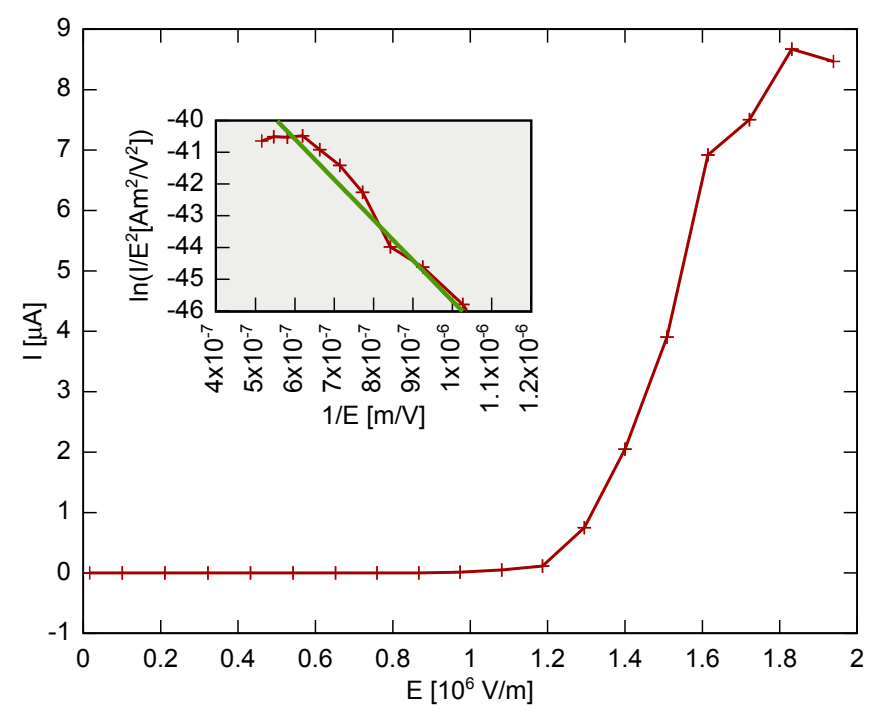

FIG. 3. Electron field emission current vs. applied field. Insert shows Fowler-Nordheim plot.

In Fig. 4, first results using CNFs to create neutrons are shown. The neutrons are generated at an ac- 
celeration voltage of $80 \mathrm{keV}$ and a emitter-target distance of $12.7 \mathrm{~mm}$. The gas flow in the chamber was $10 \mathrm{sccm}$, resulting in a pressure of $0.08 \mathrm{~Pa}$. Although the actual count rate at the detector is small (several counts/minute), a clear correlation between the applied high voltage and the neutron count rate can be seen and the signal is clearly above the background level. The measured neutron rate at the neutron detector corresponds to $2000-3000 \mathrm{n} / \mathrm{s}$ at the target, which agrees with the measured current in the low microamp range when taking into account that the target was not fully loaded with deuterium (elastic recoil detection analysis shows that our samples have a deuterium-titanium content of about 1:9 in the first $600 \mathrm{~nm}$ of the target). The achieved field is also roughly ten times higher than that required for electron emission, in agreement with values quoted in literature. Furthermore, neutron production from molecular deuterium $\left(\mathrm{D}_{2}\right)$ at these energies produces a neutron yield that is lower by a factor of ten at a current level of $1 \mu \mathrm{A}$ which would be below our detection limit.

From the Fowler-Nordheim plot for electron emission, we can also estimate that only $10^{4}$ tips contribute to the emission current. Assuming the same value for field ionization, this translates into an average current of $100 \mathrm{pA}$ per tip which is in the range of what we expect comparing to $5 \mathrm{nA}$ currents that can be achieved with highly optimized single $\operatorname{tips}^{3}$ and the gas pressures used. The process of field desorption requires a higher field than field ionization and is not expected to contribute at the local fields we achieved.

Considering the density of CNFs of $2 \times 10^{9} \mathrm{~cm}^{-2}$ and a coated area of $2 \mathrm{~cm}^{2}$, only a very low fraction $\left(10^{-5}\right)$ of tips contribute. This is a concern and we will discuss a possible solution to improve this number in the next section.



FIG. 4. Neutron yields (at the target) from the CNF sample at a deuterium gas pressure of $0.08 \mathrm{~Pa}$ and a gas cracker power of $60 \mathrm{~W}$.
High voltage breakdown occured occasionally during the experiment, but did not degrade the performance of the samples. Inspecting the samples in an SEM afterwards showed only localized damage from sparks (damaged spot size around $30 \mu \mathrm{m})$.

\section{OUTLOOK}

In ongoing experiments, we are investigating arrays of CNFs such as those shown in Fig. 5. These have the advantage of having a good separation between tips so that the field enhancement factor of a single tip is not influenced by emitters that are in close proximity and therefore a higher fraction of tips is expected to contribute to the current. In the fabrication process of these samples, one lithographic step is involved to form the array by patterning the nickel deposition. The distance between tips can be optimized and it has been found that a good value for tip height to tip separation distance is $1: 2^{10,11}$.

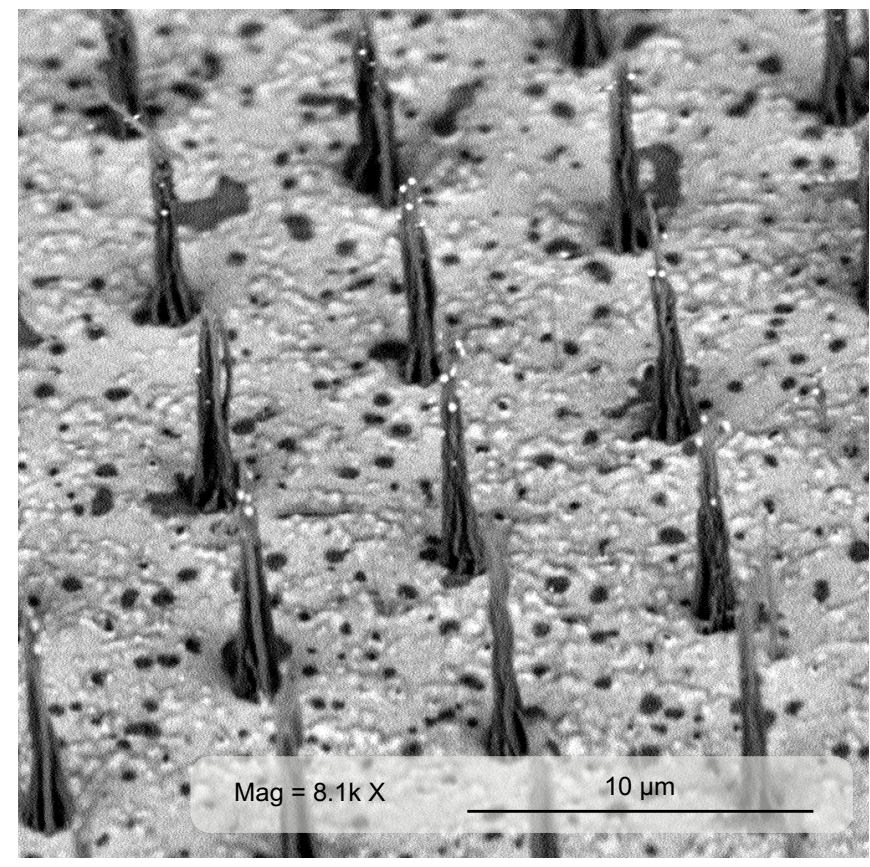

FIG. 5. Array of CNFs with a $6 \mu \mathrm{m}$ spacing using the same growth mechanism (similar growth rate and diameter) as for the CNF forest sample.

We are in the process of characterizing these new samples and have obtained electron emission results with enhancement factors of around 2000. A possible explanation for the smaller enhancement factor is that in these arrays the field is shared by several tips.

Another direction we are investigating is surfacecoating of field emitters. We envision twofold benefits: increased lifetime of the CNFs due to protection of the structure from hydrogen etching and better field emitting properties due to optimization of the work function of the emitting material. The use of different emitter 
materials, e.g., etched silicon $\operatorname{tips}^{12}$, is another option we are pursuing.

\section{CONCLUSION}

We show first results from a field ionization neutron generator. The measured field enhancement factors are comparable with those achieved using single CNFs. From this fact and the number of contributing tips calculated by Fowler-Nordheim analysis, we conclude that single CNFs that protrude from the forest of CNFs (as can be seen in the SEM images) are responsible for the field ionization current.

Previous results by Naranjo et al. ${ }^{3}$ show currents of $5 \mathrm{nA}$ for a single very well conditioned and optimized tip and they report a neutron yield of $800 \mathrm{n} / \mathrm{s}$ at $115 \mathrm{kV}$. An array of tips with a spacing appropriate to achieve high field enhancement factors should allow for $10^{6}$ tips per square centimeter, which for optimized tips should allow neutron yields in the $10^{7}-10^{8} \mathrm{n} / \mathrm{s}$ range. The few microamperes of deuterium current reported here are already sufficient for the generation of $2 \times 10^{7} \mathrm{n} / \mathrm{s}$ in a D-T reaction at $80 \mathrm{keV}$ using a fully loaded target, meeting the yield of currently used AmBe neutron sources.

For future experiments, we therefore plan to use arrays of single isolated field emitters to increase the amount of emitting tips while still generating high field enhancement factors. Furthermore, by optimizing surface conditions, we plan on increasing field ionization currents and neutron yields. We expect to be able to reach neutron yields suitable for radioactive source replacement or applications in oil-well logging.

\section{ACKNOWLEDGMENTS}

The authors would like to thank Jeff Bramble and Dave Rodgers for their help with calibrating the neutron detector, Dave Grothe for help with the simulations, and Kin Man Yu for the ERDA measurement.

This work was performed under the auspices of the US Department of Energy, NNSA Office of Nonproliferation Research and Engineering (NA-22) by Lawrence Berkeley National Laboratory under Contract DE-AC02$05 \mathrm{CH} 11231$.
* APersaud@lbl.gov

1 I. Solano, B. Reichenbach, P.R. Schwoebel, D.L. Chichester, C.E. Holland, K.L. Hertz, and J.P. Brainard, Nucl. Instrum. Methods A 587, 76 (2008).

2 B. Reichenbach, I. Solano, and P. Schwoebel, J. Appl. Phys. 103, 094912 (2008).

3 B. Naranjo, J. K. Gimzewski, and S. Putterman, Nature 434, 1115 (2005).

4 R. L. Fink, N. Jiang, L. Thuesen, K.N. Leung, A.J. Antolak and D. McDaniel, AIP Conf. Proc. CP1099, 610 (2009).

5 R. Gomer, Surf. Sci. 299-300, 129 (1994).

6 J. Csikai, Handbook of Fast Neutron Generators Volume I, CRC Press, 1987, p.74.
7 A. Friedman, D. Grote, and I. Haber, Phys. Fluids B 4, 2203 (1992).

8 M. Meyyappan, L. Delzeit, A. Cassell, and D. Hash, Plasma Sources Science and Technology 12, 205 (2003).

${ }^{9}$ R. H. Fowler and L. Nordheim, Proc. R. Soc. London. Ser. A 119, 173 (1928).

10 J.M. Bonard, N. Weiss, H. Kind, T. Stöckli, L. Forró, K. Kern, and A. Chatelain, Advanced Materials 13, 184 (2001).

11 S. H. Jo, Y. Tu, Z.P. Huang, D.L. Carnahan, D.Z. Wang, and Z.F. Ren, Appl. Phys. Lett. 82, 3520 (2003).

12 I. W. Rangelow and S. Biehl, J. Vac. Sci. Technol. B 19, 916 (2001). 
This document was prepared as an account of work sponsored by the United States Government. While this document is believed to contain correct information, neither the United States Government nor any agency thereof, nor The Regents of the University of California, nor any of their employees, makes any warranty, express or implied, or assumes any legal responsibility for the accuracy, completeness, or usefulness of any information, apparatus, product, or process disclosed, or represents that its use would not infringe privately owned rights. Reference herein to any specific commercial product, process, or service by its trade name, trademark, manufacturer, or otherwise, does not necessarily

constitute or imply its endorsement, recommendation, or favoring by the United States Government or any agency thereof, or The Regents of the University of California. The views and opinions of authors expressed herein do not necessarily state or reflect those of the United States Government or any agency thereof or The Regents of the University of California. 